\title{
Olecranon Fractures - Review of Treatment and Complications
}

\author{
Henrique Sousa*, Ricardo Oliveira, Moisés Ventura, Renato Ramos, Rita Grazina and Andreia Ferreira \\ Department of Orthopaedic and Traumatology, Centro Hospitalar Vila Nova de Gaia Espinho, Vila Nova \\ de Gaia, Portugal
}

*Corresponding author: Henrique Costa Sousa, Department of Orthopaedic and Traumatology, Centro Hospitalar Vila Nova de Gaia Espinho, Rua Francisco Sá Carneiro, s/n, 4400-129 Vila Nova de Gaia, Portugal

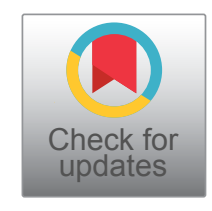

\begin{abstract}
Olecranon fractures are common upper extremity fractures usually subject to surgical fixation. Several options exist for olecranon fracture treatment, tension band, plating, intramedullary nail and fragment excision with triceps advancement are the most used but no technique is suitable for the management of all olecranon fractures and each one is associated with more or less prevalence of the most common complications, namely loss of motion, nonunion, malunion, infection, wound dehiscence, instability, symptomatic hardware, ulnar neuritis, posttraumatic arthritis, and heterotopic ossification. Overall final outcomes of olecranon fractures treatment are good, fracture union is the expected outcome regardless the osteosynthesis method and functional outcome is good to excellent in the great majority of patients.

This article reviews the treatment of olecranon fractures and its complications. For a better understanding and treatment of this fracture the elbow relevant anatomy and olecranon fracture morphology are also reviewed.
\end{abstract}

\section{Keywords}

Olecranon fractures, Complications, Functional outcomes

\section{Introduction}

Olecranon fracture is a common injury, representing 8-10\% of all elbow fractures [1], it's an intra-articular fracture and can cause disruption of the elbow extensor mechanism, therefore most of these fractures are treated surgically. There is a variety of fixation techniques, however no technique is suitable for the management of all olecranon fractures and each one is associated with more or less prevalence of the most common complications.

Injury mechanism can be a direct fall over the olecranon, which results in impaction of the olecranon against the distal humerus resulting in a comminuted fracture or a sudden eccentric triceps contraction upon a flexed elbow of a falling upper extremity, which typically results in a simple transverse or oblique fracture. Combination of the two mechanisms, especially in high energy trauma, can result in the less frequent trans-olecranon fracture dislocation which is usually associated with concomitant bony and soft tissue elbow injuries.

Most olecranon fractures are associated with good outcome, nevertheless some frequent complications can be anticipated as extension deficit, hardware irritation, and wound healing problems.

\section{Anatomy}

To correctly treat olecranon fractures we must understand its place in the proximal ulna anatomy and its importance in the normal elbow biomechanics. The elbow is a trochoid joint that consists of three articulations: The proximal radioulnar, the radiocapitellar, and the ulnotrochlear joints. Elbow stability is provided by osseous congruity and the surrounding soft tissues. Together with the coronoid process the olecranon creates the greater sigmoid notch of the ulna which articulates with the humeral trochlea allowing movement only in the flexion extension axis being therefore crucial for elbow range of motion and intrinsic stability. Both the olecranon and coronoid are elbow bony stabilizers, the olecranon prevents anterior displacement of the ulna relative to the distal humerus and the coronoid acts as a buttress to prevent posterior axial ulna translation. The medial 
collateral ligament, particularly the anterior bundle, is a primary constraint to valgus stress and the lateral ulnar collateral ligament resists varus stress and acts to prevent rotatory translation. The radial head is a secondary stabilizer against valgus and posterolateral rotational forces, it articulates with the ulna at the lesser sigmoid notch forming the proximal radioulnar joint [2]. The ulna greater sigmoid notch is all covered by articular cartilage except for a central transverse ridge known as bare area.

Triceps distal insertion comprises the posterior articular capsule and olecranon, therefore a displaced olecranon fracture represents a functional disruption of the triceps extensor mechanism.

Recent focus on the proximal ulna anatomy has demonstrated the high variability in proximal ulna morphology. In the sagittal plane $96 \%$ of patients have dorsal angulation known as the proximal ulnar dorsal angulation, which averages $5.7^{\circ}$ [3-5]. In the coronal plane the proximal ulna also has on average $14^{\circ}$ of varus angulation [6].

This anatomy is especially important with precontoured locking plates and highly comminuted fractures where these variations may difficult anatomic reconstruction. There is a strong right to left side correlation which may help to predict normal patient anatomy [3].

Lastly the subcutaneous position of the olecranon makes it vulnerable to injury and symptomatic hardware.

\section{Clinical and Radiographic Evaluation}

A complete history and physical examination are fundamental for any patient presenting with upper extremity trauma. Patients with an olecranon fracture usually present with the elbow in relative flexion supported by a splint or contralateral hand, skin abrasion at the elbow or hand may hint on the injury mechanism.

Olecranon palpation will be painful, there will be swelling and a palpable defect and displaced fragment at the fracture site may be felt. Skin should be carefully inspected for any breach. Triceps function should be evaluated by the capability of extend elbow against gravity, keeping in mind that even without a functional triceps a patient can use gravity to extend the elbow. It's also paramount to perform a vascular and neurosensory evaluation, and exclude associated injury, especially in high energy trauma.

Radiographic study must include standard anteroposterior (AP) and lateral radiographs of the elbow. A good quality true lateral radiograph is of paramount importance as it not only allows for fracture identification but also demonstrates fracture extent, degree of comminution, amount of articular surface involvement and associated elbow injuries as coronoid fracture or radial head dislocation. In the AP view painful limitation of elbow range of motion (ROM) may preclude full elbow extension in which case distal humerus may obscure osseous details of the olecranon fracture, however AP view should always be evaluated to exclude associated fractures and/or dislocations.

Computer tomography (CT scan) is useful in cases of high comminution, fracture dislocations and associated fractures, it allows the detection of intermediate articular fragments otherwise difficult to detect.

In complex fracture patterns and fracture dislocations, a contralateral elbow radiograph may be helpful in assess patient native anatomy.

\section{Classification}

There are multiple classification systems for olecranon fractures: Mayo, Colton, Schatzker, HorneTanzer, Wadsworth, AO classification system amongst others. Of these the Mayo and Schatzker classifications are the most widely used.

The Schatzker classification is based on the fracture pattern and describes 6 types of fracture - transverse, transverse impacted, oblique, comminuted, oblique distal and fracture dislocation.

The Morrey-Mayo classification is especially useful as it's simple and considers three factors which directly influence treatment decision, displacement, comminution and ulnohumeral stability. It considers three types: I undisplaced or minimally displaced, II displaced but preserved elbow stability, and III associated with elbow joint instability; each is further subdivided into A non-comminuted or B comminuted.

\section{Treatment}

The final objective of olecranon fractures treatment is to restore elbow function - functional range of motion, strength and pain-free joint movement. To achieve it our osteosynthesis goals should be anatomic articular reduction and absolute stability to allow for early articular motion and rehabilitation. Regarding anatomic reduction is of paramount importance the reconstruction of the sigmoid notch, namely the olecranon width and ulna longitudinal axis and length to allow for ulno-humeral articular congruency. Proximal radio-ulnar congruency should also be obtained and soft tissue preservation and careful handling of elbow extensor mechanism are also crucial for a successful outcome.

\section{Conservative treatment}

Few olecranon fractures in adults are amenable to nonsurgical treatment. Classically conservative treatment was reserved for undisplaced or minimally displaced fractures with intact extensor mechanism (Mayo I) and inoperable patients.

However, there is increasing evidence in favor of 
nonoperativemanagement of displaced and comminuted olecranon fractures for low demand patients with multiple comorbidities. Duckworth, et al. [7] found in their 2014 retrospective series with 43 patients with a mean age of 76 years treated conservatively with collar and cuff (35\%) and above the elbow plaster cast (65\%) a $72 \%$ rate of good and excellent short term outcome (mean 4 months) and mean Broberg and Morrey score of 83 points. The 23 surviving patients evaluated for long term outcome (mean 6 years with a minimum of 2 years) presented a mean DASH score of 2.9 points and mean Oxford elbow score (OES) of 47 points with a $91 \%$ overall patient satisfaction. In 2017 Duckworth, et al. [7] prematurely stopped a prospective randomized trial with 19 patients comparing non-operative vs. operative treatment of acute isolated displaced fractures of the olecranon in patients aged $\geq 75$ years because the rate of complications in the operative arm was considered unacceptable ( 9 out of 11 patient).

A 2019 systematic review by Lenz, et al. [8] summarized all reported cases of nonoperatively treated olecranon fractures. In their analysis with 58 patients with a mean age of 83.9 they found a mean Mayo Elbow Performance Index (MEPI) score of 95.1 and a mean DASH score of 12.3. Regarding ROM, a mean flexion of $133^{\circ}$ (range: $106-140^{\circ}$ ) was achieved. The mean extension lag was $15^{\circ}$ (range: $0-30^{\circ}$ ). Mean pain levels according the Visual Analogic Scale (VAS) was 1 (range: $0-8$ ). In total, $80.8 \%$ of patients developed fibrous nonunion of their olecranon fractures. Other complications of non operative treatment were local pain $(n=8)$ and pain during movement $(n=2)$ and clicking when moving the elbow $(n=5)$.

Despite the short follow-up periods, with consequent inconclusive information regarding the rate of posttraumatic osteoarthritis non-operative management of olecranon fractures in elderly lowdemanding patients must be considered.

Non-operative treatment involves immobilization of the elbow with a posterior splint, orthosis, or long arm cast in approximately $90^{\circ}$ of flexion for 3 weeks, followed by progressive active elbow ROM and strengthening [9].

\section{Operative treatment}

Indications for operative treatment include disruption of extensor mechanism, displaced fracture and articular incongruity. Comminuted fractures and displaced fractures distal to the midpoint of the semilunar notch should be considered unstable and also require surgical intervention. Surgical stabilization to allow for early motion, rehabilitation and return to professional activity may also be a reasonable indication for operative treatment in selected cases.

The patient is usually placed in supine with arm across chest or lateral decubitus with arm over a padded post, alternatively prone position can be used.
Tourniquet is placed in upper arm. A direct posterior longitudinal approach usually used, medial and lateral dissection allow for better fracture visualization. In case of articular comminution articular visualization is needed this can be achieved by further medial and/or lateral dissection or even a lateral approach to the joint after creation of a lateral subcutaneous flap.

Regarding the osteosynthesis construct the most used are tension band wiring and plate and screw fixation.

\section{Stable and Non-Comminuted Fractures - Mayo} IIA

Tension band construct with wires or cables in combination with two parallel Kirschner $(\mathrm{K})$ wires (1.6 $\mathrm{mm}$ ) or single cannulated partially threaded screw $(6.5 \mathrm{~mm})$ is the preferred method for this stable, non-comminuted displaced avulsion type fracture, it provides stable fixation with high union rates, it's also relatively simple and cheap.

Tension band wire (TBW) counteracts posterior tensile forces and converts them to compressive forces across the articular surface $[9,10]$.

The parallel $\mathrm{K}$ wires should be placed to the anterior cortex, this is more stable and helps to prevent wire backout and consequent soft tissue irritation comparatively to intra-medullary $\mathrm{K}$ wires. However if the wires protrude beyond the anterior cortex they may block forearm rotation or injure the anterior interosseous nerve.

Partially threaded cannulated screw is an alternative to $\mathrm{K}$ wires in a tension band construct or used in isolation, some studies suggest it may be biomechanically superior to $\mathrm{K}$ wires in terms of compression at the articular surface and resistance to gapping $[9,11]$. The screw must be of sufficient length to engage the distal intramedullary canal for adequate fixation. The usual s-shape of the proximal cubitus usually limits the use and efficiency of this method, as it may shift the fracture with screw advancement or be responsible for suboptimal distal purchase.

Plate and screw construct, usually with precontoured locking compression plate (LCP) has grown in popularity for treatment Mayo IIA fractures, plate fixation was found to be biomechanically superior both in static and dynamic stress $[10,12,13]$. Comparing the two techniques for treatment of Mayo IIA olecranon fractures a 2016 meta-analysis by Ren, et al. found no difference between the two in terms of range of motion (ROM), DASH score, improvement rate, operation time, and blood loss [14]. In 2017 Duckworth, et al. [7] reported a randomized prospective therapeutic trial comparing these two techniques in Mayo IIA and found comparable functional outcome (DASH, Broberg and Morrey, MES scores and ROM), radiographic outcome and cost; regarding complications they found 
a significantly higher incidence in the TBW group due to higher rate of removal of metalwork in symptomatic patients; loss of reduction was also twice as common in the TBW group although this difference was not statistically significant, however the most serious complications, namely infection and revision surgery occurred only in the plate group [7].

A 2018 therapeutic retrospective review by Powell, et al. concluded that plates are superior to TBW concerning post-operative morbidity, reoperation rate and cost for Mayo IIA fractures, despite there was no difference regarding function outcomes. These results were explained by the statistically significantly higher complications and reoperation rates in the TBW group, especially symptomatic hardware removal, there wasn't any complication or re-operation in the plate group which compensated for the initially higher costs [15].

Intramedullary locked nails inserted through the olecranon tip have been used with good results they allow for a minimal invasive procedure and reduce the problems of soft-tissue irritation and the subsequent need for hardware removal, biomechanically its described less gapping and loosening and higher rigidity compared with TBW, the indication for this technique is the same as TBW, Mayo IIA fractures [16].

Stable and Comminuted Fractures - Mayo IIB; and Unstable Fractures - Mayo IIIA and B

In comminuted stable fractures and those with oblique orientation a TBW can over compress the greater sigmoid notch, thereby narrowing the articular surface. In complex fracture patterns and those associated with instability TBW doesn't provide sufficient stability for fracture healing and active rehabilitation. In these cases anatomic reduction and rigid fixation through plate and screw osteosynthesis is mandatory and the actual standard of care $[1,17,18]$.

Most of the complications in the treatment of comminuted stable and unstable olecranon fractures arise from improper reduction, therefore the articular surface must be directly visualized, this can be achieved by lateral and/or medial careful dissection form the posterior incision limited by the important collateral ligaments that should be preserved, alternatively a lateral approach/window may be used to improve articular visualization, again is of paramount importance lateral collateral ligament preservation/repair. Whenever possible comminuted articular fragments should be anatomically reduced to prevent articular incongruence and narrowing of the greater sigmoid notch with consequent loss of motion and posttraumatic articular degeneration; bone graft should be used to compensate for bone loss and provide additional support if needed. Some bone loss in the non-articulating bare area is acceptable as it will fill with fibrous tissue as long as the posterior cortex is rigidly fixed [3].
A commonly used strategy is to reflect the proximal and dorsal fragments and start articular surface repair in a distal to proximal and anterior to posterior fashion using the preserved distal fragment as a keystone against which to rebuild the sigmoid notch [3].

Absolute stability should be the primary goal, therefore interfragmentary screws should be used if possible, a plate based "home run screw" for intermediate olecranon fragments has been shown to stabilize and optimize the anatomically reduced articular surface [19], a small lateral or medial plate can augment fixation when the lateral or medial cortical surface is comminuted. Although compression is desired in cases of articular comminution, over compression may lead to articular surface narrowing and may alter the radius of the semilunar notch for this reason, compression of intra-articular portions of the proximal ulna should be monitored closely intraoperatively and probably avoided in most unstable fracture patterns. Locking technology lends itself well to these situations $[3,19,20]$.

Traditional AO plating technique used a 1/3 tubular non-locking plate contoured for the proximal ulna, this evolved to pre-contoured locking compression plates. Nevertheless cadaver biomechanical studies showed no differences in stiffness or load to failure between nonlocking and locking plating [21,22]. Another important consideration is the highly variable proximal ulna anatomy, which usually includes a dorsal angulation, on average $5.7^{\circ}\left(0-14^{\circ}\right)$, this curve is not included in the pre-contoured plates and may cause malunion or alter elbow anatomical relations as radiocapitellar joint and proximal radioulnar joint $[23,24]$.

The triceps insertion must be protected, the plate can sit on top of the tendon or a longitudinal incision can be made to bury the plate and screws with posterior closure. In cases of proximal comminution or small proximal fragment suture, plate fixation may prove to be insufficient as only the most proximal screw provides fixation to this fragment and sometimes can even split it, reinforcing the plate construct with suture augmentation through the triceps tendon using a grasping stitch has been successfully reported and increases the ultimate load to failure of the plate construct compared to plate fixation alone according to cadaver biomechanical tests $[3,25,26]$.

An alternative technique for those fractures with significant comminution that preclude reconstruction and osteosynthesis is fragment excision with triceps advancement, this may prove especially useful in elderly low demand patients with severe osteopenic bone and non-united fractures. Nevertheless we should keep in mind the risk of ulno-humeral instability, therefore this technique is contraindicated in olecranon fracturedislocation, olecranon fractures associated with radial head injury or other lesions that compromise elbow stability [20,21]. After fragment excision with triceps 
advancement triceps weakness is also expected. Therefore triceps reattachment, either through bone tunnels or anchors, should balance strength and stability as dorsal reattachment maximizes triceps mechanical advantage, and alignment of the triceps tendon with the anterior surface of the sigmoid notch leads to increased stability which is useful in cases of large fragment excision and low demand patient [27]. There is no consensus in the literature regarding how much olecranon can be excised without compromise of ulno-humeral stability. An, et al. suggested that up to $50 \%$ of the olecranon can be excised, more recent biomechanical studies demonstrated that removal of as little as $12.5 \%$ of the olecranon is sufficient to alter joint stability but excision up to $75 \%$ of the olecranon doesn't leads to gross instability $[28,29]$.

Associated bone and soft tissue injuries are frequent in cases of Mayo III and some IIB olecranon fractures. These injuries usually are trans-olecranon elbow fracture dislocations and Monteggia fractures. The treatment of these associated lesion is beyond the scope of this review, nevertheless is important to remember that is of paramount importance that these lesions are all taken in consideration when planning the surgical strategy with every problem addressed to achieve a functional and stable elbow.

\section{Post-Operative Management}

Pos-operatively the elbow is splinted for pain control and soft-tissue healing for 1 to 2 weeks depending on surgeon preference and soft tissue status. The elbow may be splinted in partial or full extension to decrease tension across the fracture site. Afterwards postoperative rehabilitation for olecranon fractures is tailored to patient soft tissue status and fixation stability.

For cooperative patients in whom solid fixation was achieved, early passive and active ROM exercises may be started immediately or after the referred immobilization period. Passive motion generates less distraction at the fracture site and should be emphasized early in the rehabilitation process $[13,30]$. In cases in which stable fixation was not achieved rehabilitation must be delayed.

It's important to recall that elbow immobilization after injury, even for a period of as short as three weeks, has been shown to adversely affect the range of motion of the elbow and the functional outcome, which reinforces the importance of stable long term reliable fixation that allows early post-operative rehabilitation without fatigue failure over time [13,31].

\section{Outcome}

Fracture union is the expected outcome regardless the osteosynthesis method [1,30]. Average time to union is 3 to 4 months [32]. Functional outcome expressed by the Mayo Elbow Performance Score is good to excellent in the great majority of patients [1,30,32]. Complex fracture patterns and those with comminution especially Mayo III fractures are associated with worse short and long term outcomes [33].

A Swedish 2002 study with a mean follow-up of 19 years and a cohort of 45 women and 28 men, in which $84 \%$ had open reduction and internal fixation found a $94 \%$ good or excellent outcome, with only $12 \%$ and $4 \%$ of patients had occasional daily pain respectively. Patients lost on average of 3 ㅇ of flexion and 4응 extension. $50 \%$ of the injured elbows demonstrating radiographic signs of degeneration compared to $11 \%$ of the uninjured contralateral elbows, in fact radiographic arthrosis is common after elbow trauma but is relatively rare after olecranon fractures [34]. Nevertheless nonreconstruction of sigmoid notch dimension and contour is correlated with arthrosis [33].

If we look specifically to fixation methods there are few studies to compare TBW with plate osteosynthesis. 2017 randomised controlled trial by Duckworth, et al. [7], found comparable functional results between the two in Mayo IIA fractures with mean average range of motion at 1 year of $134^{\circ} \pm 15^{\circ}$; we could find one study where locked compression plating (LCP) outperformed TBW in 1 study, with $92 \%$ of LCP and $77 \%$ of TBW having good/excellent results, however there was no difference in motion or Disabilities of the Arm, Shoulder and Hand (DASH) score [35].

There are less studies reporting outcomes of multiplanar intramedullary fixation, in those results are similar to TBW or plate and screw fixation [36,37].

\section{Complications}

Complications after olecranon fractures include: loss of motion, nonunion, malunion, infection, wound dehiscence, instability, symptomatic hardware, ulnar neuritis, posttraumatic arthritis, and heterotopic ossification.

\section{Elbow Stiffness}

Loss of terminal extension is the most frequent complaint after olecranon fracture regardless of the treatment method, and patients should be counseled accordingly. An average loss of $10-15 \%$ compared to the contralateral side is expected in at least $75 \%$ of the patients $[33,38,39]$. Overall ROM range from near normal to $110^{\circ}$ in some studies $[36,40]$. With plates and screws fixation patients on average loose $30^{\circ}$ of ulnohumeral ROM, although some articles refer an improvement in ROM after late instrumentation removal in both TBW and plate and screw fixation $[22,41,42]$.

\section{Nonunion}

Nonunion is a rare complication occurring in approximately $1 \%$ of the cases, these are more common in higher-energy injuries, namely trans-olecranon 
fracture dislocation and Monteggia fractures. Metadiaphyseal junction is the most common site because of both limited natural vascularization and technical errors. Emphasis must be given to reduction and fixation of all bone fragments, especially intermediate fragments and also ulnar geometry to avoid intra and extra-articular nonunion [43-45].

In cases of painless, fibrous nonunion with ulnohumeral functional ROM preserved, surgical intervention is unlikely to benefit the patient. Otherwise revision surgery is indicated as bone quality quickly deteriorates, and therefore fixation will be compromised. In this setting is usual to find segmental defects and gaps of missing bone, these are especially difficult to address because shortening and gap closure is not an option as this will lead to diminution of semilunar notch radius and articular incongruity. In the existing literature it's recommended rigid fixation and bone grafting, structural if needed, which usually leads to consolidation especially if the defect is less than 4 $\mathrm{cm}$ and the and surrounded by vascularized soft tissue. Better outcomes were observed among non-unions more than $5 \mathrm{~cm}$ distal to the tip of the olecranon $[44,45]$.

In cases where this strategies fails, salvage procedures must be considered. If the proximal fragment is small, fragment excision and triceps advancement is a possibility. In cases where this results in elbow instability, total elbow arthroplasty (TEA) can be an effective salvage procedure to preserve function, nevertheless we must consider the lack of literature for use of TEA in this setting. Allograft reconstructions of the olecranon have high rates of failure [44].

\section{Malunion}

Malunions occur more commonly with comminuted fractures because of the greater risk of inadequate reduction or improper fixation.

Intra-articular malunions can lead loss of motion, rapid joint destruction and posttraumatic arthritis, so unless diagnosed before symptom appears which will allow for malunion correction, when pain appears arthrosis has already developed which forces salvage procedures like TEA or fascial interposition arthroplasty Extra-articular malunion typically occur at metadiaphyseal junction and patient presents with reduced motion or radial head subluxation, in these setting diagnosis of malunion is difficult and the loss of the usually straight dorsal ulnar cortical line should alert the surgeon of this possibility, otherwise misdiagnosis of residual posterolateral instability or an incompetent annular ligament may be the outcome [46].

In cases of precarious fixation is better to immobilize the elbow for a longer period and protect the fixation, even if that results in elbow stiffness than risk fixation failure with consequent nonunion or malunion. Beside its more predictable to surgically release a stiff elbow than to correct a malunited elbow joint [45].

\section{Symptomatic Hardware and Hardware Removal}

After extension loss, symptomatic hardware is the second most frequent complication due to the subcutaneous nature of the olecranon process. The rates of hardware removal vary in the literature and according to fixation technique.

TBW was thought to be less irritating; however, review of the literature shows rates similar or higher than plate screw fixation. Anderson, et al. [47] summarized the orthopaedic literature and found higher rate of implant removal following olecranon fractures for TBW (11\% to $82 \%$ ) compared with plating (0 to $20 \%$ ). Duckworth, et al. [7] found a higher complication rate in TBW vs. plating (63\% vs. $38 \%$ ) predominantly because of a significantly higher rate of removal of metalwork in symptomatic patients ( $50 \%$ vs. $22 \%$ ).

Intramedullary nails have the lowest hardware removal rates. There isone clinical series which registed no soft-tissue irritation and no occurrences of implant removal after use of a locked intramedullary nail at 1 year after surgery [36,37].

The rate of symptomatic hardware and its removal is probably higher than the orthopaedic surgeon perception as demonstrated by the study by Edwards, et al. [11].

\section{Instability}

Elbow instability after olecranon fixation is more common in the setting of Monteggia fractures, fragment excision and triceps advancement and with the buildup of associated bony and ligamentous injuries. In the acute setting bone excision should be avoided at all cost and proximal fragment excision reserved for low demand patients as this may contribute or lead to elbow instability [28].

It's also very important to completely diagnose and address all associated bony and ligamentous injuries as undiagnosed or untreated associated lesions may be the reason to persistent instability after anatomic olecranon reconstruction and fixation or non-anatomic reduction.

\section{Infection and Wound Dehiscence}

The elbow lacks an ample soft-tissue envelope compared to other joints. As a result, the risk of infection and wound problems should be taken seriously, especially in the olecranon where the paucity of soft tissue, renders every superficial infection a soon to be deep infection if not controlled promptly, therefore there should be a low threshold for surgical debridement. Wound healing problems are typically seen in patients with higher-energy injury mechanisms. Meticulous soft tissue handling and surgical wound 
vigilance are essential to prevent dehiscence and infection. After infection treatment one should wait and confirm infection eradication before reconstructive effort as the elbow does not tolerate multiple surgeries, and the chance for a successful outcome should not be jeopardized by impatience [44].

\section{Ulnar Nerve Neuritis}

This is an uncommon complication with prevalence ranging from $2-12 \%$ of olecranon fractures and up to $25 \%$ in fracture-dislocations. In cases of ulnar nerve palsy it's important to evaluate the possibility of medial fragments malreduction that may be compressing the nerve, in those cases this should be addressed. However most of the times it recovers with time and observation until resolution is the appropriate strategy. Electromyography timing is not well defined as function improvement can occur from 3 until 20 months. In cases which symptoms fail to improve, progressive loss or post-operative severe swelling ulnar neurolysis with or without transposition may be indicated $[18,48]$.

\section{Posttraumatic Arthrosis}

Rates of posttraumatic elbow arthrosis of up to $20 \%$ have been reported after olecranon fracture. Factors associated with higher risk of arthrosis are an articular step-off of $2 \mathrm{~mm}$ or greater and initial fracture displacement [49]. As previously noted radiographic degenerative changes can be as high as $50 \%$ of the patients after olecranon fractures but fortunately most of these are not functionally significant.

\section{Heterotopic Ossification}

Heterotopic ossification ( $\mathrm{HO}$ ) is less prevalent with olecranon fractures than elbow trauma, less than $1 \%$ in isolated olecranon fractures. In fractures of the proximal ulna and radius, there is a $7 \%$ to $37 \%$ risk of heterotopic ossification, with $20 \%$ reaching clinical significance. Involving the olecranon transolecranon fracture/dislocations are those associated with more risk of heterotopic bone formation. For simple olecranon fractures prophylaxis is not needed. In more complex injuries there is insufficient data for recommendation in favor of chemoprophylaxis with indomethacin. Radiation therapy is not recommended as it increases significantly nonunion rates [50].

\section{Conclusion}

Olecranon fractures are common fractures of the upper extremity that most frequently result from a fall from standing height. Most fractures are displaced and require surgical fixation. Preoperative planning is critical for success, Stable anatomic fixation of all fragments is essential to restore normal articular anatomy and allow for early rehabilitation. Simple fractures can be fixed with a tension band or plate and screws with crescent evidence in favor of the later; more complex fractures require plate-and-screw fixation. The most common complications are symptomatic hardware and loss of motion and patient expectations should be managed appropriately, infection should be treated aggressively. Good to excellent outcomes can be expected in the majority of patients.

\section{References}

1. Court-Brown CM, Heckman JD, McQueen (2015) Rockwood and green's fractures in adults. Wolters Kluwer Health.

2. Athwal GS, Ramsey ML, Steinmann SP, Moriatis Wolf $J$ (2011) Fractures and dislocations of the elbow: A return to the basics. Instr Course Lect 60: 199-214.

3. Rouleau DM, Sandman E, van Riet R, Galatz LM (2013) Management of fractures of the proximal ulna. J Am Acad of Orthop Surg 21: 149-160.

4. Rouleau DM, Canet F, Chapleau J, Petit Y, Sandman E, et al. (2012) The influence of proximal ulnar morphology on elbow range of motion. J Shoulder Elbow Surg 21: 384-388.

5. Beser CG, Demiryurek D, Ozsoy H, Ercakmak B, Hayran $\mathrm{M}$, et al. (2014) Redefining the proximal ulna anatomy. Surg Radiol Anat 36: 1023-1031.

6. Puchwein P, Schildhauer TA, Schoffman S, Heidari N, Windisch G, et al. (2012) Three-dimensional morphometry of the proximal ulna: A comparison to currently used anatomically preshaped ulna plates. J Shoulder Elbow Surg 21: 1018-1023.

7. Duckworth AD, Clement MD, White TO, Court-Brown CM, McQueen MM (2017) Plate versus tension band wire fixation for olecranon fractures: A prospective randomized trial. J Bone Joint Surg Am 99: 1261-1273.

8. Lenz M, Wegmann K, Müller LP, Hackl M (2019) Nonoperative treatment of olecranon fractures in the elderly-a systematic review. Obere Extremit 14: 48-52.

9. Hutchinson DT, Horwitz DS, Ha G, Thomas CW, Bachus $\mathrm{KN}$ (2003) Cyclic loading of olecranon fracture fixation constructs. J Bone Joint Surg Am 85: 831-837.

10. Wilson J, Bajwa A, Kamath V, Rangan A (2011) Biomechanical comparison of interfragmentary compression in transverse fractures of the olecranon. J Bone Joint Surg $\mathrm{Br}$ 93: 245-250.

11. Edwards SG, Cohen MS, Lattanza LL, lorio ML, Daniels C, et al. (2012) Surgeon perceptions and patient outcomes regarding proximal ulna fixation: A multicenter experience. J Shoulder Elbow Surg 21: 1637-1643.

12. Duckworth AD, Clement ND, Aitken SA, Court-Brown CM, McQueen MM, et al. (2012) The epidemiology of fractures of the proximal ulna. Injury 43: 343-346.

13. Morrey BF (2000) The Elbow and Its Disorders. ( $3^{\text {rd }}$ edn), WB Saunders, Philadelphia, USA.

14. Ren Y-M, Qiao H-Y, Wei Z-J, Lin W, Fan B-Y, et al. (2016) Efficacy and safety of tension band wiring versus plate fixation in olecranon fractures: A systematic review and meta-analysis. J Orthop Surg Res 11: 137.

15. Powell AJ, Farhan-Alanie OM, McGraw IWW (2019) Tension band wiring versus locking plate fixation for simple, two-part Mayo 2A olecranon fractures: A comparison of post-operative outcomes, complications, reoperations and economics. Musculoskelet Surg 103: 155-160. 
16. Nowak TE, Burkhart KJ, Mueller LP, Mattyasovszky SG, Andres T, et al. (2010) New intramedullary locking nail for olecranon fracture fixation-an in vitro biomechanical comparison with tension band wiring. J Trauma 69: E56-E61.

17. Buijze G, Kloen P (2009) Clinical evaluation of locking compression plate fixation for comminuted olecranon fractures. J Bone Joint Surg Am 91: 2416-2420.

18. Sahajpal D, Wright TW (2009) Proximal ulna fractures. J Hand Surg Am 34: 357-362.

19. von Rüden C, Woltmann A, Hierholzer C, Trentz O, Bühren $V(2011)$ The pivotal role of the intermediate fragment in initial operative treatment of olecranon fractures. J Orthop Surg 6: 9.

20. Prayson MJ, lossi MF, Buchalter D, Vogt M, Towers J (2008) Safe zone for anterior cortical perforation of the ulna during tension-band wire fixation: A magnetic resonance imaging analysis. J Shoulder Elbow Surg 17: 121-125.

21. lannuzzi N, Dahners L (2009) Excision and advancement in the treatment of comminuted olecranon fractures. J Orthop Trauma 23: 226-228.

22. Buijze GA, Blankevoort L, Tuijthof GJM, Sierevelt IN Kloen P (2010) Biomechanical evaluation of fixation of comminuted olecranon fractures: One-third tubular versus locking compression plating. Arch Orthop Trauma Surg 130: 459-464.

23. Argintar E, Martin BD, Singer A, Hsieh AH, Edwards S (2011) A biomechanical comparison of multidirectional nail and locking plate fixation in unstable olecranon fractures. $J$ Shoulder Elbow Surg 21: 1398-1405.

24. Quintero J (2000) In: Ruedi TP, Murphy WM, AO Principles of Fracture Management. Thieme, Stuttgart, 323-339.

25. Wild JR, Askam BM, Margolis DS, Geffre CP, Krupinski $E A$, et al. (2012) Biomechanical evaluation of sutureaugmented locking plate fixation for proximal third fractures of the olecranon. J Orthop Trauma 26: 533-538.

26. Catalano LW, Crivello K, Lafer MP, Chia B, Barron OA et al. (2011) Potential dangers of tension band wiring of olecranon fractures: An anatomic study. J Hand Surg Am 36: $1659-1662$.

27. Ferreira LM, Bell TH, Johnson JA, King GJW (2011) The effect of triceps repair techniques following olecranon excision on elbow stability and extension strength: An in vitro biomechanical study. J Orthop Trauma 25: 420-424.

28. An KN, Morrey BF, Chao EY (1986) The effect of partial removal of proximal ulna on elbow constraint. Clin Orthop Relat Res, 270-279.

29. Bell TH, Ferreira LM, McDonald CP, Johnson JA, King GJ (2010) Contribution of the olecranon to elbow stability: An in vitro biomechanical study. J Bone Joint Surg Am 92: 949-957.

30. Veillette CJH, Steinmann SP (2008) Olecranon fractures. Orthop Clin North Am 39: 229-236.

31. Scharplatz D, Allgower M (1975) Fracture-dislocations of the elbow. Injury 7: 143-159.

32. Huang T-W, Wu C-C, Fan K-F, Tseng I-C, Lee P-C, et al. (2010) Tension band wiring for olecranon fractures: Relative stability of Kirschner wires in various configurations. J Trauma 68: 173-176.

33. Rommens PM, Küchle R, Schneider RU, Reuter M (2004) Olecranon fractures in adult: Factors influencing outcome. Injury 35: 1149-1157.
34. Karlsson MK, Hasserius R, Karlsson C, Besjakov J, Josefsson P-O, et al. (2002) Fractures of the olecranon: A 15- to 25-year follow-up of 73 patients. Clin Orthop Relat Res, 205-212.

35. Schliemann B, Raschke MJ, Groene P, Weimann A, Wähnert D, et al. (2014) Comparison of tension band wiring and precontoured locking compression fixation in Mayo type IIA olecranon fractures. Acta Orthop Belg 80: 106-111.

36. Argintar E, Cohen M, Eglseder A, Edwards S (2012) Clinical results of olecranon fractures treated with multiplanar locked intramedullary nailing. J Orthop Trauma 27: 140144.

37. Gehr J, FriedI W (2006) Intramedullary locking compression nail for the treatment of an olecranon fracture. Oper Orthop Traumatol 18: 199-213.

38. Hume MC, Wiss DA (1992) Olecranon fractures. A clinical and radiographic comparison of tension band wiring and plate fixation. Clin Orthop Relat Res, 229-235.

39. Rommens PM, Schneider RU, Reuter M (2004) Functional results after operative treatment of olecranon fractures. Acta Chir Belg 104: 191-197.

40. Wang YH, Tao R, Xu H, Cao Y, Zhou Z-y, et al. (2011) Mid-term outcomes of contoured plating for comminuted fractures of the olecranon. Orthop Surg 3: 176-180.

41. Erturer RE, Sever C, Sonmez MM, Ozcelik IB, Akman $S$, et al. (2011) Results of open reduction and plate osteosynthesis in comminuted fracture of the olecranon. $J$ Shoulder Elbow Surg 20: 449-454.

42. Lindenhovius AL, Brouwer KM, Doornberg JN, Ring DC, Kloen $P$ (2008) Longterm outcome of operatively treated fracture-dislocations of the olecranon. J Orthop Trauma 22: 325-331.

43. Papagelopoulos PJ, Morrey BF (1994) Treatment of nonunion of olecranon fractures. J Bone Joint Surg $\mathrm{Br} 76$ : 627-635.

44. Baecher N, Edwards S (2013) Olecranon fractures. J Hand Surg Am 38: 593-604.

45. Rotini R, Antonioli D, Marinelli A, Katusić D (2008) Surgical treatment of proximal ulna nonunion. Chir Organi Mov 91: 65-70.

46. Jeong WK, Lee DH, Kyung BS, Lee SH (2012) Factors affecting assessment of ulnar bowing in radiography. $\mathrm{J}$ Pediatr Orthop 32: 48-53.

47. Anderson ML, Larson AN, Merten SM, Steinmann SP (2007) Congruent elbow plate fixation of olecranon fractures. $\mathrm{J}$ Orthop Trauma 21: 386-393.

48. Ishigaki $N$, Uchiyama $S$, Nakagawa $H$, Kamimura $M$, Miyasaka T, et al. (2004) Ulnar nerve palsy at the elbow after surgical treatment for fractures of the olecranon. $J$ Shoulder Elbow Surg 13: 60-65.

49. van der Linden SC, van Kampen A, Jaarsma RL (2012) $\mathrm{K}$-wire position in tension-band wiring technique affects stability of wires and longterm outcome in surgical treatment of olecranon fractures. J Shoulder Elbow Surg 21: 405-411.

50. Foruria AM, Augustin S, Morrey BF, Sánchez-Sotelo J (2013) Heterotopic ossification after surgery for fractures and fracture dislocations involving the proximal aspect of the radius or ulna. J Bone Joint Surg Am 95: e66. 